\title{
Automatic optical heterodyne phase lock by microcomputer-assisted loop filter
}

\author{
Yudai Hisata, Akira Mizutoria), and Masafumi Koga ${ }^{\text {b) }}$ \\ Oita University, 700 Dannnoharu, Oita city, Oita 870-1192, Japan
}

a)mizutori@oita-u.ac.jp

b)m.koga@oita-u.ac.jp

\begin{abstract}
This paper demonstrates signal light carrier automatic pull-in and phase-lock to an optical frequency comb reference by our newly developed microcomputer-assisted phase-lock loop circuit. The microcomputer utilizes a binary-search algorithm to alter the oscillation frequency of the slave laser and achieve rapid pull-in to the master laser. It achieves automatic phaselocking time of $0.45 \mathrm{msec}$ when the laser-diode frequency lies within the pull-in range of $\pm 100 \mathrm{MHz}$. The single side band phase noise is just $1.8^{\circ}$ at offset frequencies from $10 \mathrm{~Hz}$ to $5 \mathrm{MHz}$. This phase noise value is not expected to cause any power penalty with QPSK modulation.
\end{abstract}

Keywords: PLL, heterodyne, OFCR

Classification: Fiber-Optic Transmission for Communications

\section{References}

[1] A. Sano, M. Nagatani, H. Nosaka, and Y. Miyamoto, " $5 \times 1-\mathrm{Tb} / \mathrm{s}$ PDM16QAM transmission over $1,920 \mathrm{~km}$ using high-speed InP MUX-DAC integrated module," OFC 2015, M3G.3, Los Angeles, USA, Mar 2015.

[2] G. Raybon, J. Cho, A. Adamiecki, P. Winzer, A. Konczykowska, F. Jorge, J.-Y. Dupuy, M. Riet, B. Duval, K. Kim, S. Randel, D. Pilori, B. Guan, N. Fontaine, and E. C. Burrows, "Single carrier high symbol rate transmitter for data rates up to $1.0 \mathrm{~Tb} / \mathrm{s}$," OFC 2016, Th3A.2, Anaheim, USA, Mar 2016.

[3] S. Randel, D. Pilori, S. Corteselli, G. Raybon, A. Adamiecki, A. Gnauck, S. Chandrasekhar, P. J. Winzer, L. Altenhain, A. Bielik, and R. Schmid, "Allelectronic flexibly programmable 864-Gb/s single-carrier PDM-64-QAM," OFC 2014, PDP TH5C.8, San Francisco, USA, Mar 2014. DOI:10.1364/OFC.2014. Th5 C.8

[4] A. Carena, V. Curry, G. Bosco, P. Poggiolini, and F. Forghieri, "Modeling of the impact of nonlinear propagation effects in uncompensated optical coherent transmission links," IEEE J. Lightwave Technol., vol. 30, no. 10, pp. 15241539, May 2012. DOI:10.1109/JLT.2012.2189198

[5] OIF-TLMSA-01.0, "Multi-source agreement for CW tunable laser," May 2003.

[6] M. Koga, Y. Shigeta, F. Shirazawa, H. Ohta, and A. Mizutori, "Costas loop homodyne detection for 20-gb/s QPSK signal on the optical frequency synchronous network," IEEE J. Lightwave Technol., vol. 33, no. 23, pp. 47524760, 2015. DOI:10.1109/JLT.2015.2474713 
[7] ITU-T Recommendation G.694.1, “Spectral grids for WDM applications: DWDM frequency grid,” Feb 2012.

\section{Introduction}

The development of high-speed digital signal processors (DSP) in the last decade has enabled us to dramatically increase the spectral efficiency (SE) and transmission capacity of coherent optical communication $[1,2,3]$. DSP-based schemes can calibrate local oscillator (LO) frequency offset and estimate the relative phase between carrier and LO lights. They can also precisely compensate the linear and nonlinear fiber characteristics as well as replicate the sharp cutoff characteristics of electrical filters that yield, approximately, the Nyquist minimum bandwidth [4]. However, the SE is limited to just a single channel and in wavelength-division multiplexing (WDM) transmission, the SE degrades because of the bandwidth unnecessarily reserved to counter frequency fluctuation in each optical signal [5], even though the sharp cutoff filter can realize such dense channel spacing that the optical carrier frequency spacing matches the bandwidth of the sharp cutoff filter. Furthermore, the amount of digital signal processing is becoming a significant burden.

We have developed optical phase-lock loop (PLL) technology to realize the optical synchronous network and demonstrated stable homodyne detection for $20 \mathrm{Gbit} / \mathrm{s}$ QPSK signals [6]. If PLL technology is applied to not only long haul but also short distance optical transmission systems, we can enhance the light source frequency stability (which suppresses the unnecessary bandwidth usage), narrow its spectral line width, raise the symbol rate up to match the performance of pre-amplifiers and high-speed logic-ICs (which is not limited by analogue-to-digital conversion (ADC) speed), and reduce the DSP requirements. However, to the best of our knowledge, no paper has described the automatic pull-in and phase lock operation of slave LD frequency, which is necessary for optical communication systems.

This paper demonstrates light carrier automatic pull-in and phase-lock operation to an optical frequency grid comb reference (OFCR). The developed microcomputer-assisted (MA) analogue phase locked loop (PLL) circuit automatically pulls in and phase-locks an optical signal carrier to the OFCR. The MA-PLL starts to work by running a binary search algorithm (BSA) when the optical carrier frequency is set within $\pm 100 \mathrm{MHz}$ from some grid frequency, and locks the carrier phase to the OFCR within 0.45 milliseconds. The loop filter used in the MA-PLL is the same integral, lag and lead loop filter (I\&LL-LF) as developed for $20 \mathrm{Gbit} / \mathrm{s}$ QPSK signal homodyne detection [6], with some changes to the circuit parameter values. Once signal carrier phase locking to the OFCR commences, it achieves the single-side band phase noise of $1.8^{\circ}$ for offset frequencies from 10 to $5 \mathrm{MHz}$. 


\section{Microcomputer-assisted loop filter}

In optical heterodyne phase locking to a reference light, the loop delay is the key to realizing a low phase noise PLL. The target of the phase noise, under $2^{\circ}$, is the value that causes no symbol error, as was demonstrated in reference 6 (Ref. 6). If an optical PLL circuit is to suppress the SSB phase noise to less than $2^{\circ}$, the loop bandwidth must exceed a few megahertz (MHz) even if the light source has spectral-linewidth of the order of kHz. When enlarging the loop bandwidth to over tens of MHz for wider spectral linewidth LD sources, the analogue circuit approach is superior to DSPs because DSPs that convert analogue to digital signals suffer excessive processing delays. Thus we adopted the analogue circuit approach and used a microcomputer to enhance operation performance.

A phase-frequency detector (PFD) and integral function of loop-filter are essential to the pull in and phase-lock operation. The PFD serves to pull the slave LD frequency into the reference and the integral circuit locks its phase stably to that of the reference. However, when the initial frequency difference between the slave and reference is large, pull-in operation may not complete due to saturation of the integral circuit. If the initial frequency difference can be set to within some allowable range, the slave LD frequency could approach the reference and its phase is locked by controlling its injection current.

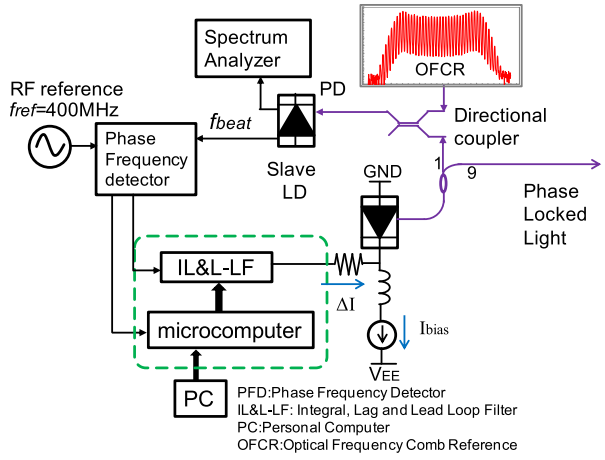

(a) MA-PLL circuit

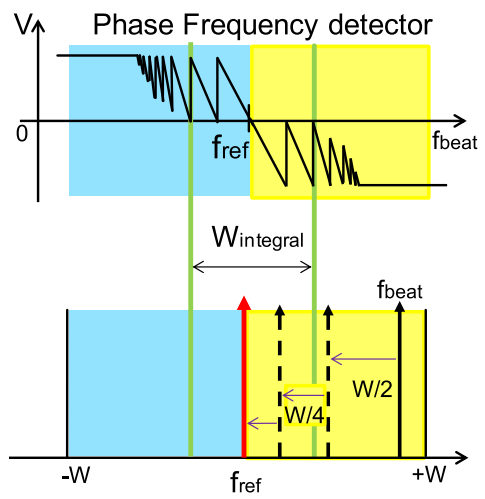

(c) PFD's characteristics and optical frequency behavior

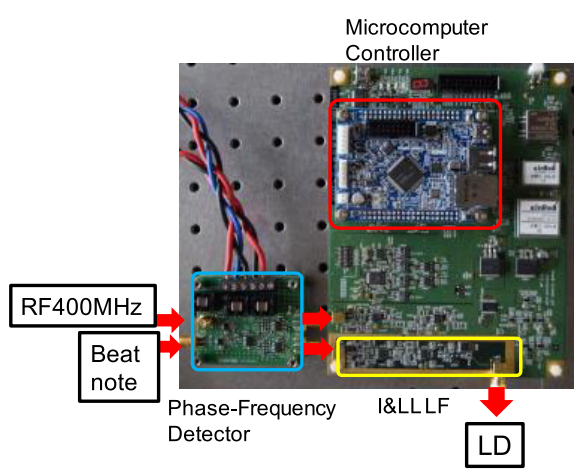

(b) Photograph of MA-PLL prototype

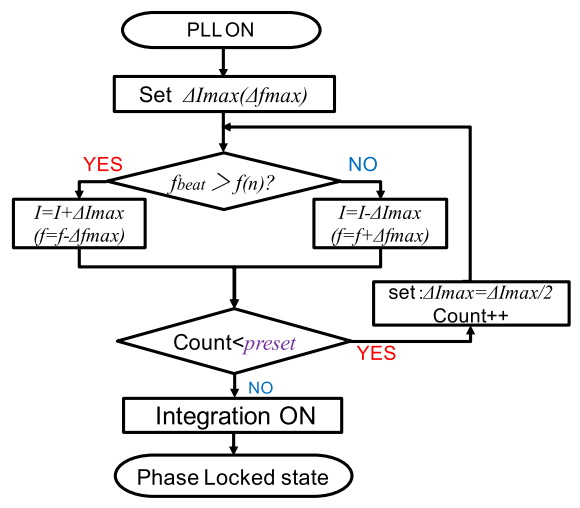

(d) Binary search algorithm
() IEICE 2016

DOI: $10.1587 /$ comex.2016XBL0152 Received August 24, 2016 Accepted September 1, 2016 Publicized September 20, 2016 Copyedited December 1, 2016
Fig. 1. Microcomputer-assisted phase-lock loop and its binary search algorithm. 
Fig. 1(a) shows the MA-PLL circuit with a photograph of our prototype in (b). The MA-PLL consists of a 32-bit, $96 \mathrm{MHz}$ clock, microcomputer, I\&LL-LF, PFD, photo-diode (PD) followed with trans-impedance amplifier (TIA), and an LD that emits the optical signal carrier. The OFCR in Fig. 1(a) provides the ITU-T standardized grid frequencies of $f(n)=193.1 \mathrm{THz}+25 \mathrm{GHz} \times n$; where, $n$ is integer from -15 to 15 [7]. The red solid rectangle in Fig. 1(b) indicates the microcomputer, the yellow the I\&LL loop filter, and the blue the PFD. The PD detects the beat frequency, $f_{\text {beat }}$, between the OFCR and the LD lights, and after comparing it with a $400 \mathrm{MHz}$ RF reference, its phase error is extracted via the I\&LL-LF. By feeding back the phase error information to the LD injection current, $I_{\text {bias }}$, the LD oscillation frequency of the light carrier can be phase-locked to the OFCR, where, $I_{\text {bias }}$ corresponds to $f_{\text {beat }}$.

The MA-PLL circuit starts to work when the slave LD frequency is set in a range of $\pm \mathrm{W}_{\text {ini }}$; where, $\mathrm{W}_{\text {ini }}$ is the maximum pull-in range specified for the MAPLL. The PFD output is monitored and when $f(n)\left(=f_{\text {ref }}\right)<f_{\text {beat }}$, the PFD outputs negative voltage while it outputs positive when $f_{\text {beat }}<f(n)$, as shown in Fig. 1(c). Here, the BSA starts with the maximum variation of $\Delta I_{\max }$ at the initial injection current value of $I_{\text {bias (0) }}$; where, $\Delta I_{\max }$ corresponds to $\mathrm{W}_{\text {ini }} / 2$. The microcomputer sets $I_{\text {bias }}$ as $I_{\text {bias (I) }}=I_{\text {bias (0) }}-\Delta I_{\text {max }}$ if $f(n)<f_{\text {beat }}$ and $I_{\text {bias }(0)}+\Delta I_{\text {max }}$ otherwise, as shown in Fig. 1(d). Again the microcomputer monitors the PFD, and resets $I_{\text {bias }}$ to $I_{\text {bias }(2)}=\left(I_{\text {bias }(0)}-\Delta I_{\max }\right)+\Delta I_{\max } / 2$ if $f_{\text {beat }}$ becomes less than $f(\mathrm{n})$ and $\left(I_{\text {bias }(0)}+\Delta I_{\max }\right)-\Delta I_{\max } / 2$ if $f_{\text {beat }}$ becomes larger than $f(\mathrm{n})$; where, $I_{\text {bias }(i)}$ indicates the $i^{\text {th }}$ modified injection current. This process is repeated until the count number reaches a preset value. In our design, $\mathrm{W}_{\text {ini }}=100 \mathrm{MHz}$ and $W_{\text {integral }}=$ $30 \mathrm{MHz}$, thus the preset value is set to be $3\left(100 \mathrm{MHz} / 2^{3-1}<30 \mathrm{MHz}\right)$. Upon discovering $I_{\text {bias }}(3)$, the microcomputer activates the integral circuit in the I\&LL loop filter, which automatically phase-locks the slave LD to the reference.

\section{Demonstration of automatic phase-locking operation}

We examined the automatic pull-in and phase-lock operation of our proposal. The LD used was an external-cavity-structured LD (E-LD) identical to the one used in Ref. 6. Its FM response is $-20 \mathrm{MHz} / \mathrm{mA}$ to $-2.8 \mathrm{MHz} / \mathrm{mA}$ between $\mathrm{DC}$ and $1 \mathrm{MHz}$, as shown in Fig. 2(a). The optical frequency pull-in operation was monitored by utilizing our optical frequency discrimination circuit that had been developed for $20 \mathrm{Gbit} / \mathrm{s}$ QPSK homodyne detection, see Ref. 6, as shown in Fig. 2(b). The optical frequency discrimination circuit is comprised of a 12.5 GHz-spaced arrayed-waveguide grating (AWG), two pin-photodiodes followed by trans-impedance amplifiers and a differential amplifier. The set of electrical amplifiers forms a balanced amplifier that exhibits a common-mode rejection ratio (CMRR) of over $43 \mathrm{~dB}$ for a measurement bandwidth between $\mathrm{DC}$ to $10 \mathrm{MHz}$. The AWG's transmittance has a Gaussian profile of

$$
f(x)=\exp \left\{-\frac{\left(x \pm x_{0}\right)^{2}}{2 \sigma^{2}}\right\}
$$

where, $x_{0}=12.5 / 2=6.25 \mathrm{GHz}$ and $\sigma=5.31 \mathrm{GHz}$ for the AWG used. The AWG crossover optical frequency was thermally controlled to $f(0)=193.1 \mathrm{THz}$, and the 
transmittance of two adjacent ports separated by $12.5 \mathrm{GHz}(3 \mathrm{~dB}$ down) is as shown in Ref. 6. The discrimination sensitivity at crossover frequency was $1.4 \mathrm{MHz} / \mathrm{mV}$ for AWG input optical power of $6.0 \mathrm{dBm}$. The circuit output voltage for a frequency shifts of $100 \mathrm{MHz}$ was $71 \mathrm{mV}$. Note that the common mode amplitude component, triggered by the injection current variation, was completely suppressed due to the circuit's CMRR and we could extract the true optical frequency behavior of the light emitted by the LD.

Before processing the BSA, we set the LD optical frequency at about $\pm 100 \mathrm{MHz}$ from $f(0)$ of OFCR by monitoring the beat frequency. After frequency setup, the phase-lock loop was turned on and confirmed automatic pull-in and phase-lock operation. The results are shown in Fig. 2(b) and (c).

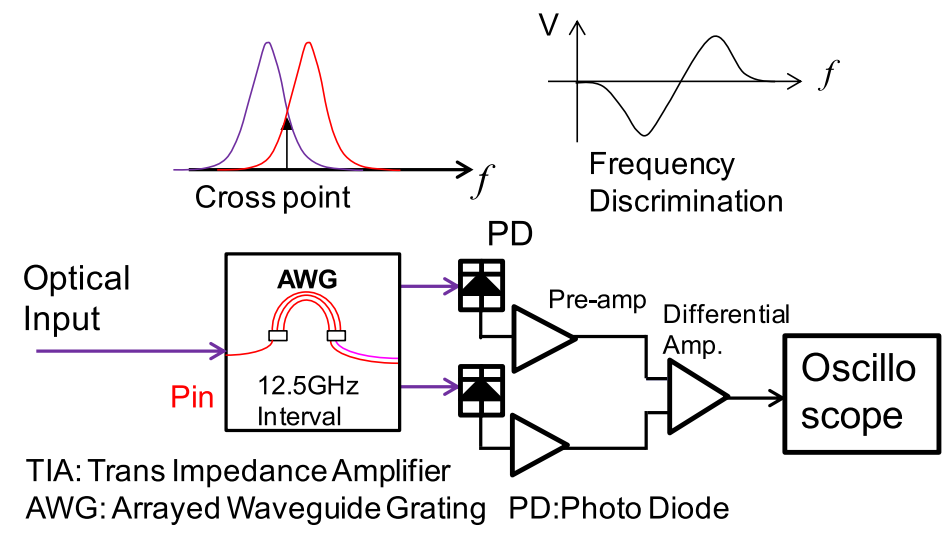

(a) Schematic diagram of frequency discrimination

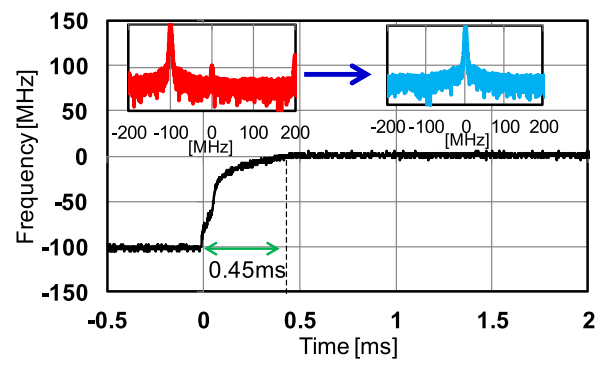

(b) Start at $-100 \mathrm{MHz}$ from the reference

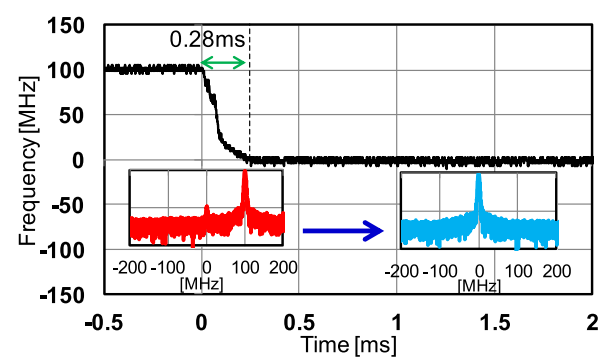

(c) Start at $+100 \mathrm{MHz}$ from the reference

Fig. 2. Measurement results for pull-in operation.

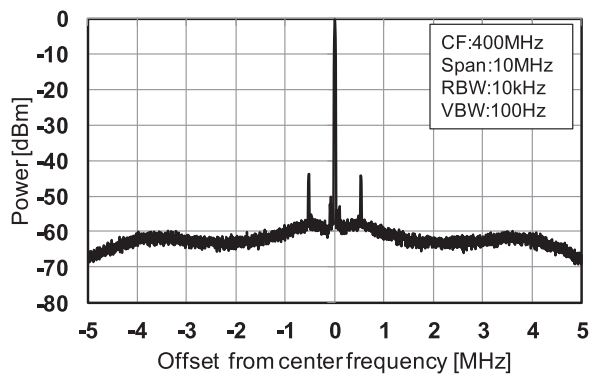

(a) RF spectrum

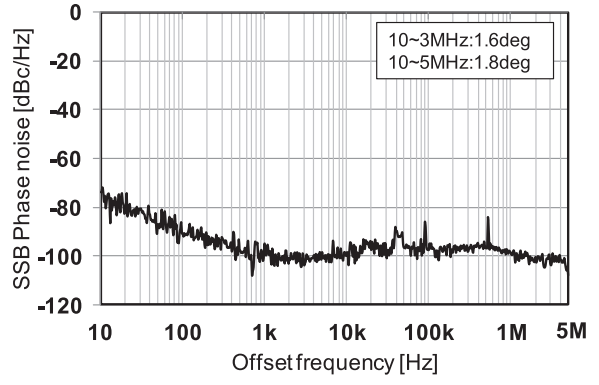

(b) SSB phase noise

Fig. 3. Measured RF spectrum and SSB phase noise. 
When $f_{\text {beat }}$ was set at $-100 \mathrm{MHz}$ from the reference microwave frequency of $400 \mathrm{MHz}$ (see the inset spectrum of Fig. 2(b)), the discriminator output showed that the optical frequency automatically shifted from $-100 \mathrm{MHz}$ to 0 in $0.45 \mathrm{~ms}$. Setting $f_{\text {beat }}$ at $+100 \mathrm{MHz}$, as shown in Fig. 2(c), the optical frequency shifted from $100 \mathrm{MHz}$ to 0 in $0.28 \mathrm{~ms}$. After transition, we confirmed the phase-lock state by observing the RF spectrum. The measured RF spectrum and SSB phase noise are shown in Fig. 3(a) and (b), respectively. We can see $60 \mathrm{~dB}$ beat note power to noise level at around the center frequency $(=400 \mathrm{MHz})$ and a smooth noise floor spreading to $3 \mathrm{MHz}$. This noise floor indicates a $3 \mathrm{MHz}$ PLL bandwidth. Note that the spur at $0.5 \mathrm{MHz}$ offset frequency observed in the beat spectrum is crosstalk from the digital thermo controller for E-LD. SSB phase noise has a standard deviation of only $1.6^{\circ}$ and $1.8^{\circ}$ for offset frequencies from $10 \mathrm{~Hz}$ to $3 \mathrm{MHz}$ and $5 \mathrm{MHz}$, respectively (Agilent Technologies: N9010A), as shown in Fig. 3(b).

These results confirm that our developed MA-PLL circuit achieves automatic pull-in and phase-lock operation.

\section{Conclusions}

We successfully demonstrated signal light carrier automatic pull-in and phase-lock operation by our newly developed microcomputer-assisted phase-lock loop circuit. It achieves automatic phase-lock within $0.45 \mathrm{~ms}$ when the laser-diode frequency lies within the pull-in range of $\pm 100 \mathrm{MHz}$. The achieved phase-lock state exhibited SSB phase noise of just $1.8^{\circ}$ at $5 \mathrm{MHz}$ offset frequency.

\section{Acknowledgments}

The work is partly supported by National Institute of Information and Communication Technology (NICT) Japan and JSPS KAKENHI Grant Number 15H04009. 